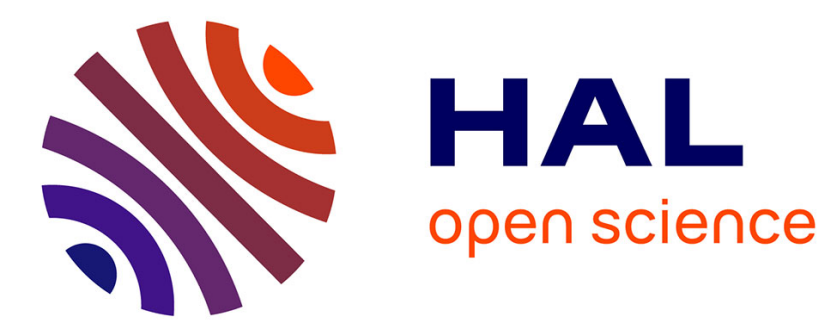

\title{
Isomorphisms of the De Bruijn digraph and free-space optical networks
}

\author{
David Coudert, Afonso Ferreira, Stéphane Pérennes
}

\section{To cite this version:}

David Coudert, Afonso Ferreira, Stéphane Pérennes. Isomorphisms of the De Bruijn digraph and freespace optical networks. Networks, 2002, 40 (3), pp.155 - 164. 10.1002/net.10043 . inria-00429201

\section{HAL Id: inria-00429201 https://hal.inria.fr/inria-00429201}

Submitted on 1 Nov 2009

HAL is a multi-disciplinary open access archive for the deposit and dissemination of scientific research documents, whether they are published or not. The documents may come from teaching and research institutions in France or abroad, or from public or private research centers.
L'archive ouverte pluridisciplinaire HAL, est destinée au dépôt et à la diffusion de documents scientifiques de niveau recherche, publiés ou non, émanant des établissements d'enseignement et de recherche français ou étrangers, des laboratoires publics ou privés. 


\title{
Isomorphisms of the De Bruijn Digraph and Free-Space Optical Networks
}

\author{
D. Coudert A. Ferreira S. Perennes \\ MASCOTTE Project - CNRS-I3S-INRIA \\ 2004, route des Lucioles - BP 93 \\ F-06902 Sophia-Antipolis - France \\ \{David.Coudert, Afonso.Ferreira, Stephane.Perennes\}@sophia.inria.fr
}

\begin{abstract}
The de Bruijn digraph $B(d, D)$ has degree $d$, diameter $D, d^{D}$ vertices and $d^{D+1}$ arcs. It is usually defined by words of size $D$ on an alphabet of cardinality $d$, through a cyclic left shift permutation on the words, after which the rightmost symbol is changed. In this paper, we show that any digraph defined on words of a given size, through an arbitrary permutation on the alphabet and an arbitrary permutation on the word indices, is isomorphic to the de Bruijn digraph, provided that this latter permutation is cyclic. We use this result to improve from $O\left(d^{D+1}\right)$ to $\Theta\left(\sqrt{d^{D+1}}\right)$ the number of lenses required for the implementation of $B(d, D)$ by the Optical Transpose Interconnection System proposed by Marsden et al. (Optics Letters 18(13):1083-1085, July 1993).

Keywords: Distributed systems, Optical networks, Free space of optical interconnections, Optical Transpose Interconnection System (OTIS), de Bruijn digraph, Graph isomorphisms.
\end{abstract}




\section{Introduction}

Many results describing technological and theoretical advances in optical network topologies and design exist in the literature. In this paper, we give optimal optical layouts of several de Bruijn-like topologies, using existing optical technologies, namely the Optical Transpose Interconnecting System (OTIS) architecture proposed by Marsden et al. in [17]. These results are obtained using graph isomorphisms developed here.

Our motivation stems from the fact that it has been shown that the break-even line length is less than $1 \mathrm{~cm}$, when optical communication lines become more effective than their electrical counterparts, in terms of speed and power consumption [13]. Therefore, the use of optical interconnections between processors on the same board is justified, and some studies even suggest that on-chip optical interconnects will soon be cost effective [22]. Moreover, the emergence of cutting-edge technologies such as Vertical Cavity Surface-Emitting Lasers (VCSELs), high sensibility optical transimpedance receivers, beam splitters, micro-lenses and holograms, makes possible the fabrication of complex optical communication networks $[18,22]$

The OTIS architecture is a simple means of implementing very dense one-to-one interconnections between processors in a free space of optical interconnections [17]. In a nutshell, it consists of two lenslet arrays allowing a large number of optical interconnections from a set of transmitters to a set of receivers as shown in Figure 1. The OTIS architecture is used by the 3-D OptoElectronic Stacked Processors consortium to connect a 16-by-16 switch (for more details, see http://soliton.ucsd.edu/3doesp/public/).

It has been shown [23] that the OTIS architecture can be used to build all-optical complete 


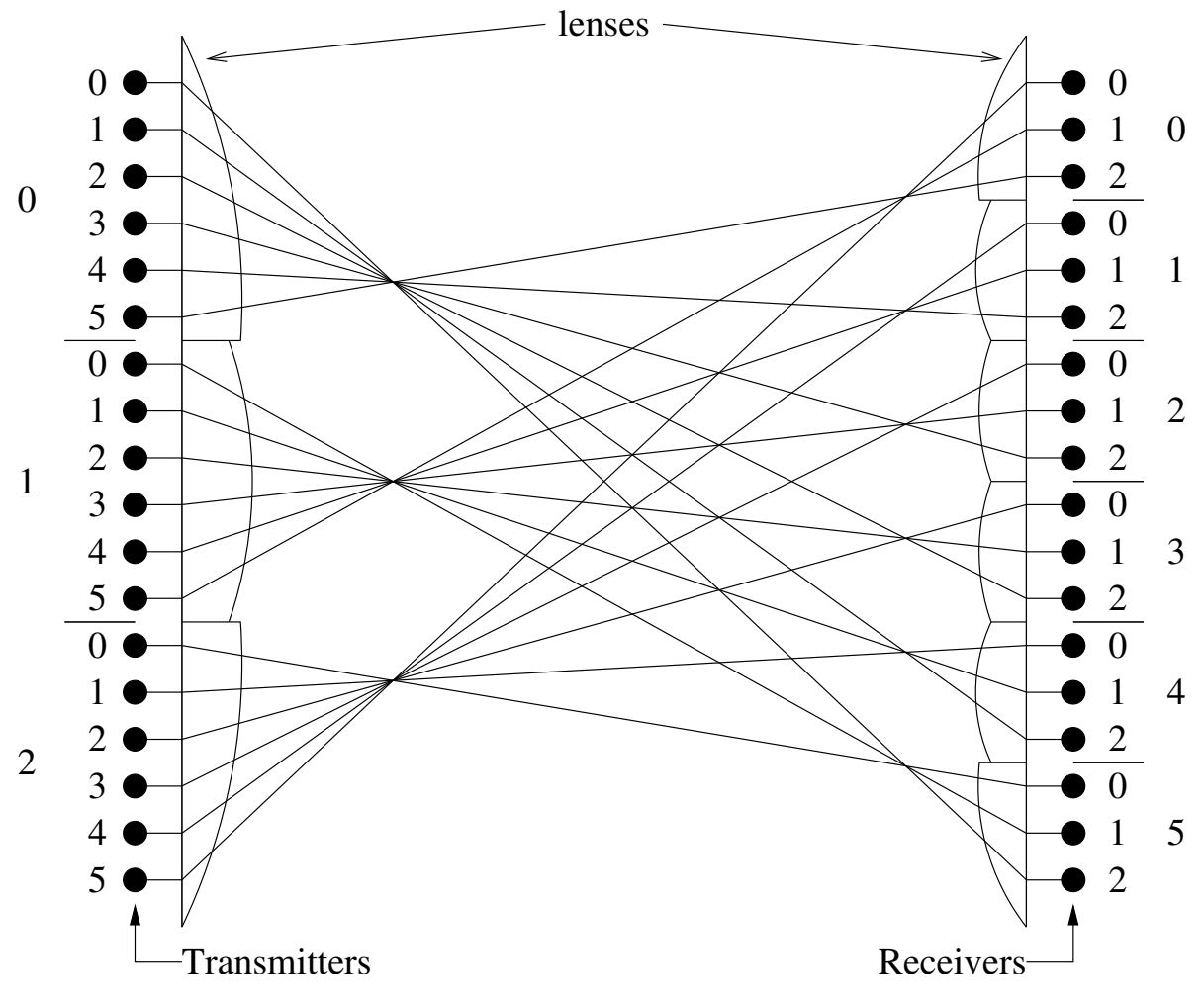

Figure 1: $\operatorname{OTIS}(3,6)$. 
networks based on a symmetric complete digraph with loops $\left(K_{n}^{+}\right)$. The OTIS architecture and recent progress made in optoelectronics allow the implementation of $K_{64}^{+}$by OTIS, in which each processor has 64 transceivers (one per neighbor in $K_{64}^{+}$). It has also been shown [11] how to implement the single-hop multi-OPS POPS network [7], and the multi-hop multiOPS stack-Kautz network [10], with the OTIS architecture. However, these layouts do not scale well, since they require a large number of transceivers per processor, as well as a large number of optical lenses. Indeed, the efficiency of an OTIS layout is often measured by the number of lenses required. Consequently, an important research direction is to study the set of network topologies for which there are lens-efficient layouts with the OTIS architecture.

In our work, we focus on de Bruijn-like digraphs, since the de Bruijn digraph [1] is well known for its applications in parallel and distributed computing [6] and it has been extensively studied in graph theory [12]. It has also been used to build various multi-stage interconnection networks [18], and, more interestingly, the Galileo space probe of NASA uses a de Bruijn network with 8192 vertices to implement a signal decoder [8]. A de Bruijn digraph $B(d, D)$ is an alphabet digraph [1] of constant degree $d$ and diameter $D$. It has for vertices the $d^{D}$ words of length $D$ on the alphabet $Z_{d}=\{0,1, \ldots, d-1\}$, and a vertex $x=x_{D-1} \ldots x_{1} x_{0}$, $x_{i} \in Z_{d}$, is adjacent to the set of vertices $\Gamma^{+}(x)=\left\{x_{D-2} \ldots x_{1} x_{0} \beta, \beta \in Z_{d}\right\}$.

From a theoretical point of view, we show in this paper that there are many ways to build digraphs which are isomorphic to the de Bruijn digraph. We first show, in Proposition 3.2, that the alphabet digraph $B_{\pi}(d, D)$, built by using a permutation $\pi$ on $Z_{d}$ and the adjacency relation $\Gamma_{B_{\pi}(d, D)}^{+}(x)=\left\{\pi\left(x_{D-2}\right) \ldots \pi\left(x_{1}\right) \pi\left(x_{0}\right) \pi(\beta), \beta \in Z_{d}\right\}$, is isomorphic to $B(d, D)$. This result yields a new and simple proof of the isomorphism between the de Bruijn digraph and 
the Imase-Itoh digraph [14]. Then, we show, in Theorem 3.8, that the alphabet digraph $A(f, \pi, j)$, built by using a permutation $f$ on $Z_{D}$, a permutation $\pi$ on $Z_{d}$ and the adjacency relation $\Gamma_{A(f, \pi, j)}^{+}(x)=\left\{\pi\left(x_{f(D-1)}\right) \ldots \pi\left(x_{f(j+1)}\right) \pi(\beta) \pi\left(x_{f(j-1)}\right) \ldots \pi\left(x_{f(1)}\right) \pi\left(x_{f(0)}\right), \beta \in Z_{d}\right\}$, is isomorphic to $B(d, D)$ if and only if $f$ is cyclic. Notice that the permutation $\pi$ is applied on the alphabet and $f$ is applied on the word indices (see Section 3.2). Finally, we show that the permutations $\pi$ and $f$ may be applied together to characterize a class of digraphs isomorphic to the de Bruijn digraph.

It has been shown in [11] that the OTIS architecture can implement the Imase and Itoh digraph $I I(d, n)$ with $n$ vertices of degree $d$. Our result on the isomorphism of $I I(d, n)$ and $B(d, D)$ implies that OTIS can also implement the de Bruijn. Unfortunately, for a fixed degree $d$, such a layout uses $O(d n)$ lenses to interconnect $n$ processors, and it is not very efficient. Our subsequent results help us to show how OTIS can implement the de Bruijn digraph with only $\Theta(\sqrt{d n})$ lenses, which is not only optimal, but also very efficient for practical values, as the number of lenses determines also their size.

This paper is organized as follows. In Section 2, we give the graph theoretical machinery needed in the remainder. Then, in Section 3, we show that there are many isomorphisms of the de Bruijn digraph, using first a permutation $\pi$ on the alphabet $Z_{d}$ and then a permutation $f$ on the indices $Z_{D}$. In fact, there are exactly $d !(D-1)$ ! such isomorphic digraphs. The application to optical networks is given in Section 4, where we present OTIS layouts of de Bruijn-like digraphs and we show how to obtain such layouts where the minimum number of lenses is used. We close the paper with some concluding remarks and directions for further research. 


\section{Preliminaries}

\section{$2.1 \quad$ Notation}

Let $Z_{n}=\{0,1,2, \ldots, n-1\}$ denote the ring of integers modulo $n, Z_{n}^{k}$ the $k$ dimensional vector space over $Z_{n}$ and $e_{0}, e_{1}, \ldots, e_{k-1}$ the canonical basis. Addition of elements of $Z_{n}$ will always be performed in $Z_{n}$ (i.e., modulo $n$ ).

For any $\alpha \in Z_{n}$, let $\alpha . e_{j}$ denote the vector $(\overbrace{0, \ldots, 0}^{j}, \alpha, 0 \ldots, 0)$. Furthermore, let $Z_{n} . e_{j}$ denote the set of vectors $\left\{\alpha \cdot e_{j}, \alpha \in Z_{n}\right\}$. We define the sum of two sets of vectors as $A+B=\{a+b, a \in A, b \in B\}$. Note that $Z_{a} \cdot e_{i}+Z_{a} \cdot e_{j}=\left\{\alpha \cdot e_{i}+\beta . e_{j}, \alpha, \beta \in Z_{a}\right\} \neq$ $Z_{a} \cdot\left(e_{i}+e_{j}\right)=\left\{\alpha \cdot\left(e_{i}+e_{j}\right), \alpha \in Z_{a}\right\}$.

For a permutation $f$ and an integer $i$, we define $f^{i}$ inductively by taking $f^{0}$ as the identity permutation and $f^{i+1}=f \circ f^{i}$.

The next permutation will be used frequently.

Definition 2.1 Let $\mathcal{C}$ denote the complement permutation in $Z_{n}$ defined by $\mathcal{C}(u)=n-u-1$. $\mathcal{C}(u)$ will also be denoted $\bar{u}$.

\subsection{Classical Digraphs}

Given a digraph $G=(V, A)$, with vertex set $V$ and arc set $A$, and a vertex $x \in V$, we will denote by $\Gamma_{G}^{+}(x)$ the set of the out-neighbors of $x$, i.e. $\{y \in V,(x, y) \in A\}$. When the digraph $G$ is clear from the context we simply denote it by $\Gamma^{+}(x)$.

Definition 2.2 ([1]) The de Bruijn digraph $B(d, D)$ of degree $d$ and diameter $D$ is defined as a digraph on the set of vertices made of words of length $D$ on $Z_{d}$, and such that for 
$x=x_{D-1} x_{D-2} \cdots x_{1} x_{0}, \Gamma^{+}(x)=\left\{x_{D-2} \cdots x_{1} x_{0} \beta, \beta \in Z_{d}\right\}$.

Definition 2.3 The conjunction $G_{1} \otimes G_{2}$ of two digraphs $G_{1}=\left(V_{1}, A_{1}\right)$ and $G_{2}=\left(V_{2}, A_{2}\right)$ is the digraph with vertex set $V_{1} \times V_{2}$, where there is an arc from $\left(u_{1}, u_{2}\right)$ to $\left(v_{1}, v_{2}\right)$ if and only if there is both an arc from $u_{1}$ to $v_{1}$ in $G_{1}$ and an arc from $u_{2}$ to $v_{2}$ in $G_{2}$.

Alternatively we have $\Gamma_{G_{1} \otimes G_{2}}^{+}\left(\left(u_{1}, u_{2}\right)\right)=\Gamma_{G_{1}}^{+}\left(u_{1}\right) \times \Gamma_{G_{2}}^{+}\left(u_{2}\right)$. Also, note that $B(d, k) \otimes$ $B\left(d^{\prime}, k\right)=B\left(d d^{\prime}, k\right)$; one can see $[3,21]$ for more details about conjunction, de Bruijn digraphs and line digraphs.

Definition $2.4([\mathbf{1 9}, \mathbf{2 0}])$ The Reddy-Pradhan-Kuhl digraph $R P K(d, n)$ of degree d with $n$ vertices, is the digraph with vertex set $Z_{n}$ and such that for $u \in Z_{n}$ :

$\Gamma^{+}(u)=\{d u+\alpha(\bmod n), 0 \leq \alpha<d\}$.

The digraphs $R P K\left(d, d^{D}\right)$ and $B(d, D)$ are isomorphic (see [19]). To see this, we associate with vertex $x=x_{D-1} x_{D-2} \cdots x_{1} x_{0}$ of $B(d, D)$ a number $u \in Z_{n}$ with $u=\sum_{i=0}^{D-1} x_{i} d^{i}$; then the out-neighbors of $x$ in $B(d, D)$ are associated with numbers of the form $d u+\alpha, 0 \leq \alpha<d$. Note also that this congruence definition is sometimes considered as the standard one for the de Bruijn digraph.

Definition 2.5 ([16]) The Kautz digraph $K(d, D)$ of degree $d$ and diameter $D$ is defined as a digraph on the set of vertices made of words of length $D$ on the alphabet $Z_{d+1}$, such that $x=x_{D-1} \cdots x_{1} x_{0}$, with $x_{i} \in Z_{d+1}$ and $x_{i} \neq x_{i+1} . \Gamma^{+}(x)=\left\{x_{D-2} \cdots x_{1} x_{0} \beta, \beta \neq x_{0}, \beta \in\right.$ $\left.Z_{d+1}\right\}$. 
Definition 2.6 ([14]) The Imase and Itoh digraph $I I(d, n)$ of degree $d$ with $n$ vertices is the digraph with vertex set $Z_{n}$ and such that for $u \in Z_{n}: \Gamma^{+}(u)=\{-d u-\alpha(\bmod n), 1 \leq$ $\alpha \leq d\}$

It has been shown [15] that $I I\left(d, d^{D-1}(d+1)\right)$ is isomorphic to $K(d, D)$.

\section{$3 \quad B(d, D)$ as a digraph on an alphabet}

In this section we define generalizations of the de Bruijn digraph. In order to obtain the out-neighbors of a vertex labeled by some word of length $D$ over $Z_{d}$ we proceed as follows:

- instead of performing a left-shift on the word letters we permute the letter positions in some arbitrary way;

- then each letter is replaced by another one according to some fixed permutation on the alphabet $Z_{d}$;

- finally the letter of a given fixed position is replaced by an arbitrary letter of $Z_{d}$.

\subsection{Permutation on the alphabet}

We start by considering only the case of a permutation on the alphabet. We will show that there is an isomorphism between $B(d, D)$ and digraphs that we call $B_{\pi}(d, D)$, i.e., which are similar to $B(d, D)$ up to a permutation on the alphabet $Z_{d}$. Then we will prove that $I I\left(d, d^{D}\right)$ and $B(d, D)$ are isomorphic, which, according to previous remarks will imply that $I I\left(d, d^{D}\right), B(d, D), R P K\left(d, d^{D}\right)$ are isomorphic. 
Definition 3.1 Given a permutation $\pi$ on $Z_{d}, B_{\pi}(d, D)$ is defined as the digraph on the set of vertices made of words of length $D$ on $Z_{d}$, such that for each vertex $x=x_{D-1} x_{D-2} \cdots x_{1} x_{0}$, $x_{i} \in Z_{d}, \Gamma^{+}(x)=\left\{\pi\left(x_{D-2}\right) \cdots \pi\left(x_{1}\right) \pi\left(x_{0}\right) \beta, \beta \in Z_{d}\right\}$.

Proposition $3.2 B(d, D)$ and $B_{\pi}(d, D)$ are isomorphic.

Proof: We just have to define an isomorphism $W$ from $B_{\pi}(d, D)$ to $B(d, D)$; $W$ is the following one-to-one mapping from the words of length $D$ on the alphabet $Z_{d}$ onto itself: $W\left(x_{D-1} x_{D-2} \ldots x_{1} x_{0}\right)=\pi^{0}\left(x_{D-1}\right) \pi^{1}\left(x_{D-2}\right) \ldots \pi^{D-2}\left(x_{1}\right) \pi^{D-1}\left(x_{0}\right)$.

Given a vertex $x$ of $B_{\pi}(d, D)$ we have

$$
\begin{aligned}
W^{-1} \Gamma_{B(d, D)}^{+}\left(W\left(x_{D-1} x_{D-2} \ldots x_{0}\right)\right) & =W^{-1} \Gamma_{B(d, D)}^{+}\left(\pi^{0}\left(x_{D-1}\right) \pi^{1}\left(x_{D-2}\right) \ldots \pi^{D-1}\left(x_{0}\right)\right) \\
& =W^{-1}\left(\pi^{1}\left(x_{D-2}\right) \ldots \pi^{D-2}\left(x_{1}\right) \pi^{D-1}\left(x_{0}\right) \beta\right), \beta \in Z_{d} \\
& =\pi\left(x_{D-2}\right) \ldots \pi\left(x_{0}\right) \pi(\beta) \\
& =\pi\left(x_{D-2}\right) \ldots \pi\left(x_{0}\right) \alpha, \alpha \in Z_{d} .
\end{aligned}
$$

As the last line corresponds to the adjacency relation in $B_{\pi}(d, D)$ we have defined the required isomorphism.

Notice that more generally, the digraph of degree $d$, diameter $D$ and $n=d^{D}$ vertices such that $\Gamma^{+}\left(x_{D-1} x_{D-2} \ldots x_{1} x_{0}\right)=\left\{\pi_{0}\left(x_{D-2}\right) \pi_{1}\left(x_{D-3}\right) \ldots \pi_{D-2}\left(x_{0}\right) \pi_{D-1}(\alpha), \alpha \in Z_{d}\right\}$, where $\pi_{i}$, $i \in Z_{D}$, is a permutation on $Z_{d}$, is isomorphic to $B(d, D)$.

Proposition 3.3 The digraphs $B(d, D)$ and $I I\left(d, d^{D}\right)$ are isomorphic.

Proof: Let $\bar{B}(d, D)$ denote $B_{\mathcal{C}}(d, D)$, where $\mathcal{C}$ is the complement permutation over $Z_{d}$. As in the case of $B(d, D), \bar{B}(d, D)$ can be alternatively defined as a digraph with vertex set $Z_{n}$ 
in which for $u \in Z_{n}, \Gamma^{+}(u)=\{-(d u+\beta)-1,0 \leq \beta<d\}$, that is $\Gamma^{+}(u)=\{-d u-\alpha, 1 \leq$ $\alpha \leq d\}$. This is the definition of $I I\left(d, d^{D}\right)$. So $B_{\mathcal{C}}(d, D)$ and $I I\left(d, d^{D}\right)$ are isomorphic. From Proposition 3.2 these digraphs are hence isomorphic to $B(d, D)$.

We thus obtain

Corollary 3.4 The digraphs $B(d, D), R P K\left(d, d^{D}\right)$ and $I I\left(d, d^{D}\right)$ are isomorphic.

To illustrate, Figure 2 shows the digraph $B(2,3)$ which is isomorphic to $R P K(2,8)$ of Figure 3 and to $I I(2,8)$ represented in Figure 4.

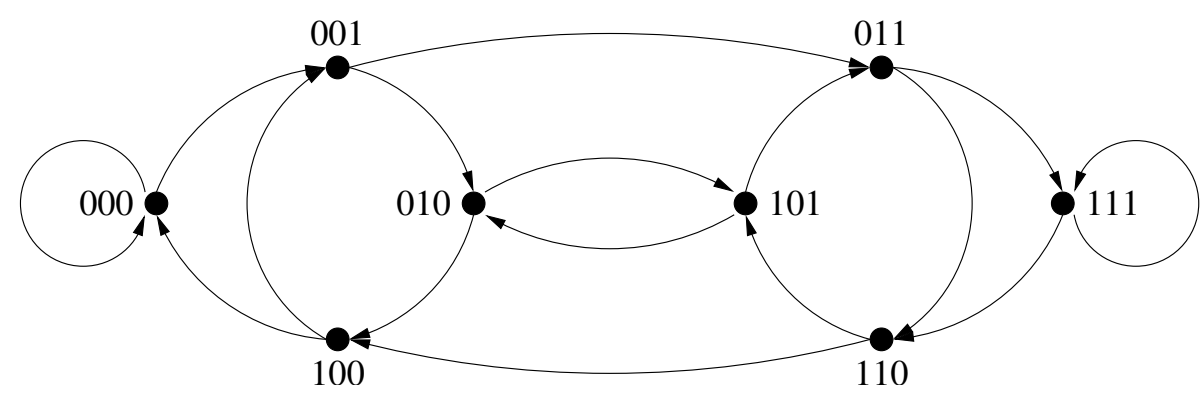

Figure 2: de Bruijn digraph of degree 2, diameter 3 and 8 vertices.

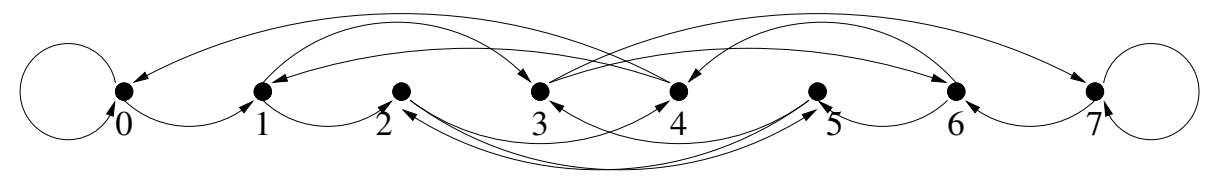

Figure 3: RPK digraph of degree 2, diameter 3 and 8 vertices.

\subsection{Permutation on the indices, digraphs $A(f, \pi, j)$}

Now we consider more general digraphs than $B_{\pi}(d, D)$ for which the left shift is replaced by any permutation on $Z_{D}$. First we need some definitions. 


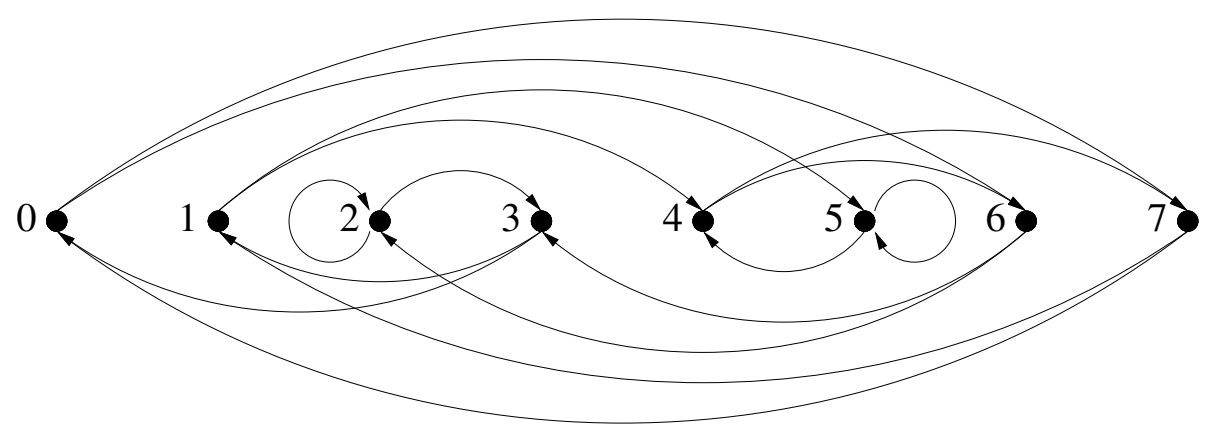

Figure 4: II digraph of degree 2, diameter 3 and 8 vertices.

Definition 3.5 To a permutation $f$ on $Z_{D}$, we associate the linear map $\vec{f}$ of $Z_{d}^{D}$ defined on the basis by $\vec{f}\left(e_{i}\right)=e_{f(i)}$ (this linear map is associated with a permutation matrix). Note that $\overrightarrow{f g}=\vec{f} \vec{g}$.

Definition 3.6 Any permutation $\pi$ on $Z_{d}$ is extended naturally to $Z_{d}^{D}$ by setting for $x=$ $x_{D-1} x_{D-2} \cdots x_{1} x_{0}: \pi(x)=\pi\left(x_{D-1}\right) \pi\left(x_{D-2}\right) \cdots \pi\left(x_{1}\right) \pi\left(x_{0}\right)$.

Definition 3.7 Let $d, D \in N$. Given a permutation $f$ on $Z_{D}$, a permutation $\pi$ on $Z_{d}$ and a position $j \in Z_{D}$, we define the digraph $G=A(f, \pi, j)$ by

$$
\begin{aligned}
V(G) & =Z_{d}^{D} \\
\Gamma_{G}^{+}(x) & =\pi(f(x))+Z_{d} \cdot e_{j}
\end{aligned}
$$

$A(f, \pi, j)$ has degree $d$ and we will say that it has dimension $D$.

Note that vertices of $B(d, D)$ can be considered as elements of $Z_{d}^{D}$ (by associating $x_{D-1} \cdots x_{1} x_{0}$ with $\left.\sum x_{i} \cdot e_{i}\right)$. Let $\sigma$ be the permutation on $Z_{D}$ defined by $\sigma: i \longmapsto i+1($ $\bmod D$ ), and let $I d$ be the identity permutation on $Z_{d}$. Then the de Bruijn digraph is exactly $A(\sigma, I d, 0)$. Indeed for $x=x_{D-1} x_{D-2} \cdots x_{1} x_{0}, \Gamma_{A(\sigma, I d, 0)}^{+}(x)=\left\{x_{D-2} \cdots x_{1} x_{0}\left(x_{D-1}+\gamma\right), \gamma \in\right.$ $\left.Z_{d}\right\}=\left\{x_{D-2} \cdots x_{1} x_{0} \beta, \beta \in Z_{d}\right\}$. Similarly, $B_{\pi}(d, D)$ and $A(\sigma, \pi, 0)$ are isomorphic. 
Theorem 3.8 The digraph $G=A(f, \pi, j)$ with degree $d$ and dimension $D$ is isomorphic to $B(d, D)$ if and only if $f$ is a cyclic permutation on $Z_{D}$.

Proof: We will prove the theorem in two steps. First, we show that when $f$ is a cyclic permutation on $Z_{D}$, then the digraphs $G=A(f, \pi, j)$ and $B(d, D)$ are isomorphic. Then, we show, by contradiction, that if the digraphs $G=A(f, \pi, j)$ and $B(d, D)$ are isomorphic then $f$ is a cyclic permutation on $Z_{D}$.

Case 1: Suppose that $f$ is a cyclic permutation on $Z_{D}$. We show that the digraphs $A(f, \pi, j)$ and $B(d, D)$ are isomorphic.

Note that since $f$ is a cyclic permutation it induces a unique orbit on $Z_{D}$. We associate with $f$ the unique permutation $g$ on $Z_{D}$, such that $\forall i \in Z_{D}, g(i)=f^{i}(j)$. From this definition, we have

$$
\begin{aligned}
& g^{-1} f g(i)=g^{-1} f f^{i}(j)=g^{-1} f^{i+1}(j)=g^{-1} g(i+1)=i+1 \\
& \left.g^{-1}(j)=0 \quad \text { hence } \overrightarrow{g^{-1}}\left(e_{j}\right)=e_{0}\right)
\end{aligned}
$$

Let us consider the digraph isomorphism induced by $\vec{g}$. For $x \in Z_{d}^{D}$, we compute $\overrightarrow{g^{-1}} \Gamma_{G}^{+}(\vec{g}(x))$. From the definition of $G, \Gamma_{G}^{+}(x)=\pi(\vec{f}(x))+Z_{d} \cdot e_{j}$, thus by linearity $\overrightarrow{g^{-1}} \Gamma_{G}^{+}(\vec{g}(x))=\pi\left(\overrightarrow{g^{-1}}(\vec{f} \vec{g}(x))\right)+Z_{d} \cdot \overrightarrow{g^{-1}}\left(e_{j}\right)$. But from (1), $\overrightarrow{g^{-1} f g}=\vec{\sigma}$ and from $(2), \overrightarrow{g^{-1}}\left(e_{j}\right)=e_{0}$. Finally, $\overrightarrow{g^{-1}} \Gamma_{G}^{+}(\vec{g}(x))=\pi(\vec{\sigma}(x))+Z_{d} \cdot e_{0}$, and this adjacency relation is the one of $B_{\pi}(d, D)$ which is isomorphic to $B(d, D)$.

Case 2: Suppose now that the digraphs $A(f, \pi, j)$ and $B(d, D)$ are isomorphic and let us show that $f$ is a cyclic permutation on $Z_{D}$.

Suppose, by contradiction, that $f$ is not a cyclic permutation on $Z_{D}$. Then it induces 
at least two orbits on $Z_{D}$. Let $\operatorname{orb}(j)$ be the set of indices sharing the orbit of $j, \operatorname{orb}(j)=$ $\left\{i \in Z_{D}, \exists k<D, f^{k}(j)=i\right\}$. Let $f=f_{\text {orb }(j)} \circ f_{r(j)}$, where $f_{\text {orb }(j)}$ is a permutation on $Z_{D}$ such that $f_{\text {orb }(j)}$ is a cyclic permutation on $\operatorname{orb}(j)$ and is equal to the identity permutation on $r(j)=Z_{D} \backslash \operatorname{orb}(j)$. Let also $f_{r(j)}$ be a permutation on $Z_{D}$ such that $f_{r(j)}$ is a permutation on $r(j)$ and is equal to the identity permutation on $\operatorname{orb}(j)$.

Then, $f^{|o r b(j)|}(j)=\left(f_{\text {orb }(j)} \circ f_{r(j)}\right)^{|\operatorname{orb}(j)|}(j)=f_{\text {orb }(j)}^{|\operatorname{orr}(j)|} \circ f_{r(j)}^{|\operatorname{orb}(j)|}(j)=f_{\text {orb }(j)}^{|\operatorname{orb}(j)|}(j)=j$.

Now, we shall consider separately the case where $|\operatorname{orb}(j)| \leq D-2$, and the case where $|\operatorname{orb}(j)|=D-1$.

A. Let $|\operatorname{orb}(j)| \leq D-2$. We show that the digraph $A(f, \pi, j)$ is not connected.

Let $x$ be a vertex of $A(f, \pi, j), x=x_{D-1} x_{D-2} \cdots x_{1} x_{0}=\sum_{i \in o r b(j)} x_{i} \cdot e_{i}+\sum_{k \in r(j)} x_{k} \cdot e_{k}$, and let $X=\left\{\sum_{i \in o r b(j)} Z_{d} \cdot e_{i}+\sum_{k \in r(j)} y \cdot e_{k}, y \in Z_{d}\right\}=\left\{\sum_{i \in o r b(j)} Z_{d} \cdot e_{i}+Z_{d} \cdot \sum_{k \in r(j)} e_{k}\right\}$. Note that $|X|=d^{|\operatorname{orb}(j)|}$, and since $|\operatorname{orb}(j)| \leq D-2$, we have $X \subsetneq Z_{d}^{D}$.

Computing the neighborhood of $X$ yields

$$
\begin{aligned}
\Gamma_{G}^{+}(X) & =\pi(\vec{f}(X))+Z_{d} \cdot e_{j} \\
& =\pi\left(\vec{f}\left(\sum_{i \in \text { orb }(j)} Z_{d} \cdot e_{i}+Z_{d} \cdot \sum_{k \in r(j)} e_{k}\right)\right)+Z_{d} \cdot e_{j} \\
& =\sum_{i \in \text { orb }(j)} \pi\left(Z_{d}\right) \cdot \vec{f}_{\text {orb }(j)}\left(e_{i}\right)+\pi\left(Z_{d}\right) \cdot \sum_{k \in r(j)} \vec{f}_{r(j)}\left(e_{k}\right)+Z_{d} \cdot e_{j} \\
& =\sum_{i \in \text { orb }(j) j} \pi\left(Z_{d}\right) \cdot e_{i}+Z_{d} \cdot e_{j}+Z_{d} \cdot \sum_{k \in r(j)} e_{k} \\
& =\sum_{i \in \text { orb }(j)} Z_{d} \cdot e_{i}+Z_{d} \cdot \sum_{k \in r(j)} e_{k} \\
& =X
\end{aligned}
$$

Hence, $X$ and $Z_{d}^{D} \backslash X$ are not connected, which implies that $A(f, \pi, j)$ is not connected 
when $|\operatorname{orb}(j)| \leq D-2$, and thus that $A(f, \pi, j)$ cannot be isomorphic to $B(d, D)$.

B. Let $|\operatorname{orb}(j)|=D-1$. We show that $A(f, \pi, j)$ cannot be isomorphic to $B(d, D)$ if $\pi$ is a cyclic permutation on $Z_{d}$, and that, otherwise, $A(f, \pi, j)$ is not connected.

Let $Y_{l}=\left\{\sum_{i \in o r b(j)} Z_{d} \cdot e_{i}+l . e_{k}, k=Z_{d} \backslash \operatorname{orb}(j)\right\}, l \in Z_{d}$. We have $\Gamma_{G}^{+}\left(Y_{l}\right)=Y_{\pi(l)}$.

(i) When $\pi$ is a cyclic permutation on $Z_{d}$, it follows that $\pi(l) \neq l$, and thus $\forall l \in$ $Z_{d}, Y_{l} \cap Y_{\pi(l)}=\emptyset$. Therefore, $A(f, \pi, j)$ has no loops, implying that it cannot be isomorphic to $B(d, D)$.

(ii) When $\pi$ is not a cyclic permutation on $Z_{d}$, we have $\cup_{1 \leq s \leq d} \pi^{s}(l) \subsetneq Z_{d}$. Hence, $\bigcup_{1 \leq s \leq d} \Gamma_{G}^{+s}\left(Y_{l}\right)=\bigcup_{1 \leq s \leq d} Y_{\pi^{s}(l)} \subsetneq Z_{d}^{D}$ and $A(f, \pi, j)$ is not connected.

Notice that since there are $(D-1)$ ! cyclic permutation functions $f$ on $Z_{D}$, Theorem 3.8 leads to $(D-1)$ ! alternative ways of defining $B(d, D)$. Furthermore, as Proposition 3.2 leads to $d$ ! other ways of defining $B(d, D)$, using permutations $\pi$ on $Z_{d}$, we finally obtain $d$ ! $(D-1)$ ! alternative ways of defining $B(d, D)$.

If $f$ is not a cyclic permutation on $Z_{D}$, it can be shown that each connected component of $A(f, \pi, j)$ is the conjunction of a de Bruijn digraph with a circuit. Furthermore, in case $A(f, \pi, j)$ is connected, and $d=D-1$, then the digraph $A(f, \pi, j)$ is isomorphic to the wrapped Butterfly digraph $W B F(d, D-1)$ of degree $d$ and $D-1$ levels with $d^{D-1}$ vertices each. See $[2,9]$ for more details. 


\subsection{Examples}

The following examples illustrate Theorem 3.8. First, we show an alphabet digraph $G=$ $A(f, I d, 2)$ which is isomorphic to $B(d, 6)$. Then we show an alphabet digraph $H=A(f, I d, 1)$ where the permutation $f$ is not cyclic and the orbit of 1 has size $D-2$, and hence $H$ is not connected.

\subsection{1 $G=A(f, I d, 2)$}

Let $G$ be the digraph with degree $d$, dimension 6 and vertex set $Z_{d}^{6}$, defined below:

$$
\Gamma_{G}^{+}\left(x_{5} x_{4} x_{3} x_{2} x_{1} x_{0}\right)=x_{2} x_{1} x_{0} \beta x_{5} x_{4} \text {, with } \beta \in Z_{d}
$$

$G$ is indeed $A(f, I d, 2)$, where $f$ is the following permutation on $Z_{6}$ :

$$
f(i)= \begin{cases}i+3 & \text { if } i<3 \\ 2 & \text { if } i=3 \\ i+2(\bmod 6) & \text { otherwise. }\end{cases}
$$

Then, $\vec{f}$ is defined on $Z_{d}^{6}$ by

$$
\vec{f}\left(x_{5} x_{4} x_{3} x_{2} x_{1} x_{0}\right)=x_{2} x_{1} x_{0} x_{3} x_{5} x_{4}
$$

The permutation $g$ associated with $f$, used in Theorem 3.8 (i.e., $g(i)=f^{i}(2)$ ), is such that $g(0)=2, g(1)=5, g(2)=1, g(3)=4, g(4)=0, g(5)=3$, and $\vec{g}\left(x_{5} x_{4} x_{3} x_{2} x_{1} x_{0}\right)=$ $x_{1} x_{3} x_{5} x_{0} x_{2} x_{4}$, and hence $\overrightarrow{g^{-1}}\left(x_{5} x_{4} x_{3} x_{2} x_{1} x_{0}\right)=x_{3} x_{0} x_{4} x_{1} x_{5} x_{2}$. See Figure 5 for an illustration. 


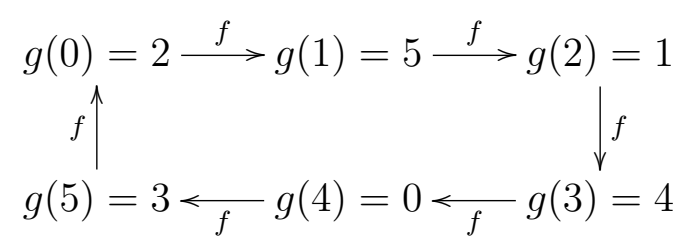

Figure 5: Illustration of the permutation $g$ defined as $g(i)=f^{i}(2)$.

Then

$$
\begin{aligned}
\overrightarrow{g^{-1}} \Gamma_{G}^{+} \vec{g}\left(x_{5} x_{4} x_{3} x_{2} x_{1} x_{0}\right) & =\overrightarrow{g^{-1}} \Gamma_{G}^{+}\left(x_{1} x_{3} x_{5} x_{0} x_{2} x_{4}\right) \\
& =\overrightarrow{g^{-1}}\left(x_{0} x_{2} x_{4} \beta x_{1} x_{3}\right), \beta \in Z_{d} \\
& =x_{4} x_{3} x_{2} x_{1} x_{0} \beta \\
& =\Gamma_{B(d, 6)}^{+}\left(x_{5} x_{4} x_{3} x_{2} x_{1} x_{0}\right)
\end{aligned}
$$

Therefore, $G$ is isomorphic to $B(d, 6)$.

\subsection{2 $H=A(f, I d, 1)$}

Let $H$ be the digraph with degree $d$, dimension 3 , and vertex set $Z_{d}^{3}$ defined below:

$$
\Gamma_{H}^{+}\left(x_{2} x_{1} x_{0}\right)=x_{0} \beta x_{2}, \text { with } \beta \in Z_{d}
$$

$H$ is indeed $A(f, I d, 1)$, where $f$ is the permutation on $Z_{3}$ such that $f(i)=\bar{i}=2-i$. Then, $\vec{f}$ is defined on $Z_{d}^{3}$ by $\vec{f}\left(x_{2} x_{1} x_{0}\right)=x_{0} x_{1} x_{2}$.

If we define the function $g$ associated with $f$ by $g(i)=f^{i}(1)$, we get $g(0)=g(1)=g(2)=$ 1 ; hence $g$ is not a cyclic permutation on $Z_{3}$. Furthermore, the orbit induced by $j=1$ is $\operatorname{orb}(j)=\{1\}$ with $|\operatorname{orb}(j)|=1 \leq D-2=1$ and thus $H$ is not connected. Notice that the digraph $H$ can be alternatively defined by

$$
\Gamma_{H}^{+}\left(\left[x_{2} x_{0}\right],\left[x_{1}\right]\right)=\left\{\left[x_{0} x_{2}\right],[\beta]\right\}
$$


It is composed of $\frac{d^{2}-d}{2}$ digraphs $C_{2} \otimes B(d, 1)$ plus $d$ digraphs $C_{1} \otimes B(d, 1)$, as shown in Figure 6 for $d=2$.
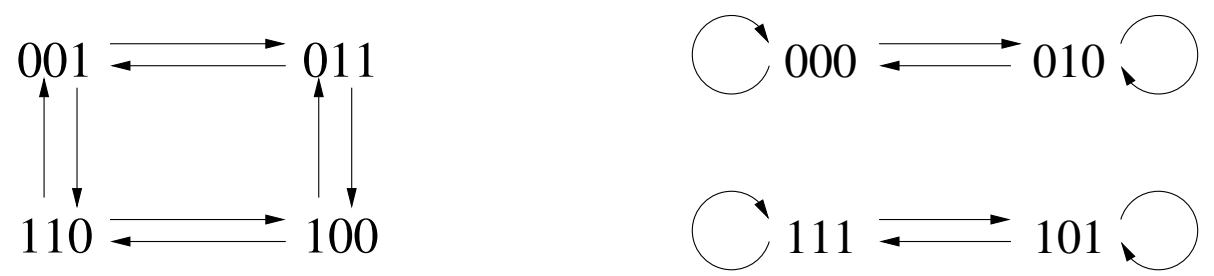

Figure 6: The digraph $H$ of Example 3.3.2, when $d=2$.

\section{OTIS layout for digraphs}

In this section, we first review the definition of the OTIS architecture, and the known digraphs having a layout with OTIS. Then, we study the particular case of the OTIS layout of de Bruijn digraphs, and, using the theoretical results developed in the previous section, we show how to obtain optimal OTIS layouts for the de Bruijn digraphs.

\subsection{The OTIS architecture}

$\operatorname{OTIS}(p, q)$ is a free-space optical system which allows one-to-one communications from $p$ groups of $q$ transmitters to $q$ groups of $p$ receivers, using $p+q$ lenses. This architecture connects the transmitter $(i, j), 0 \leq i \leq p-1,0 \leq j \leq q-1$, to the receiver $(q-j-1, p-i-1)$. Optical interconnections in the OTIS architecture are realized with a pair of lenslet arrays [4] in a free space of optical interconnections as shown in Figure 1. 


\subsection{Known OTIS layouts}

Let $m=p q$. The OTIS $(p, q)$ architecture connects $m$ transmitter units of the processors to $m$ receiver units of the same set of processors. The interconnection network can be defined as follows. Given a degree $d$ such that $d$ divides $m$, let $H(p, q, d)$ be the $d$-regular digraph with $n=$ $\frac{m}{d}$ vertices and vertex set $Z_{n}$, in which the $d$ transmitters $(\lfloor(d u+\alpha) / q\rfloor, d u+\alpha(\bmod q))$, $\alpha \in Z_{d}$, and the $d$ receivers $(\lfloor(d u+\alpha) / p\rfloor, d u+\alpha(\bmod p)), \alpha \in Z_{d}$, belong to the vertex $u \in Z_{n}$ (i.e., a processing unit). There is a connection from vertex $u$ to vertex $v$ in $H(p, q, d)$ if one transmitter of $u$ is connected to one receiver of $v$. For instance, Figure 7 represents the digraph $H(4,8,2)$. A formal definition of $H(p, q, d)$ is as follows.

Definition 4.1 The digraph $H(p, q, d)$ is defined from the OTIS $(p, q)$ architecture. It has degree $d$, such that $d$ divides $p q$, and $n=\frac{p q}{d}$ vertices and $m=p q$ arcs. It has vertex set $V(H)=Z_{n}$, and its neighborhood is defined as follows:

$$
\Gamma_{H}^{+}(u)=\left\{v=\left\lfloor\frac{(p q-1)\left(\left\lfloor\frac{d u+\alpha}{q}\right\rfloor+1\right)-p(d u+\alpha)}{d}\right\rfloor, \quad 0 \leq \alpha \leq d-1\right\}
$$

Definition 4.2 A digraph $G=(V, E)$, with $|V|=n$ vertices of constant degree $d$ and $|E|=m=d n$ arcs, has an OTIS $(p, q)$-layout if and only if there exist $p$ and $q$, with $p q=m$, and an isomorphism from $G=(V, E)$ onto $H(p, q, d)$.

Let $G^{-}$be the digraph obtained by reversing all the arcs of a digraph $G$. We remark that if $G$ admits an $\operatorname{OTIS}(p, q)$-layout then $G^{-}$has an $\operatorname{OTIS}(q, p)$-layout.

It has been proved that the Imase and Itoh digraph $I I(d, n)$ with $n$ vertices of degree $d$ has an OTIS $(d, n)$-layout [11], and consequently, so do the de Bruijn and the Kautz digraphs by Proposition 3.3. For fixed degree $d$, such a layout uses $O(d n)$ lenses to interconnect 
$n$ processors, which is not very efficient. In the following we will show how to build an OTIS $(p, q)$-layout for the de Bruijn digraph, such that the number $p+q$ of lenses used is $\Theta(\sqrt{d n})$, which is also very efficient for practical values. Note that the values of $p$ and $q$ determine the size of the lenses and, by technological considerations, it is preferable to work with lenses of almost equal size (i.e., $p \sim q$ ).

\subsection{The degree-diameter problem for OTIS}

For given degree $d$ and diameter $D$, one can study the maximum number of vertices of the family of connected digraphs $H(p, q, d)$ (i.e., the $d$-regular digraph built from an $O T I S(p, q))$. We implemented a simple and exhaustive search, based on a computer program which returns the diameter of any input digraph $H(p, q, d)$ (in case $H$ is connected). We executed this program for all pairs $(p, q)$, such that $\frac{p q}{d} \leq \sum_{i=0}^{D_{m}} d^{i}=\frac{d^{D_{m}+1}-1}{d-1}$, which is the Moore bound for degree $d$ digraphs of maximum diameter $D_{m}[5]$. The results obtained are reported in Table 1, for degree 2 and diameters 8, 9, and 10. Note that the table contains only the largest digraphs found for each diameter.

We remark that the Kautz digraph appears to be the largest digraph of degree $d$ and diameter $D$ which has an $O T I S(p, q)$-layout. This would not be surprising, since it is among the best known constructions for the classic degree-diameter problem [5].

On the other hand, for fixed degree $d$, diameter $D$, and number of vertices $n=d^{D}$, we found several digraphs $H(p, q, d)$, with $p q=d^{D+1}$ for different values of $p$ and $q$ such that both $p$ and $q$ were powers of $d$. All these digraphs happened to be isomorphic to $B(d, D)$. As an example, $H(2,256,2), H(4,128,2)$ and $H(16,32,2)$ are isomorphic to $B(2,8)$. In the next 
section, we will prove that whenever $D$ is even and $H(p, q, d)$, as defined above, is connected, then it is isomorphic to $B(d, D)$. We will also address the case where $D$ is odd.

\begin{tabular}{|c|c|c|}
\hline $\mathrm{n}$ & $\mathrm{p}$ & $\mathrm{q}$ \\
\hline$\vdots$ & $\vdots$ & $\vdots$ \\
\hline 253 & 2 & 253 \\
\hline 254 & 2 & 254 \\
\hline 255 & 2 & 255 \\
\hline 256 & 2 & 256 \\
\cline { 2 - 3 } & 4 & 128 \\
\cline { 2 - 3 } & 16 & 32 \\
\hline 258 & 2 & 258 \\
\hline 264 & 2 & 264 \\
\hline 288 & 2 & 288 \\
\hline 384 & 2 & 384 \\
\hline
\end{tabular}

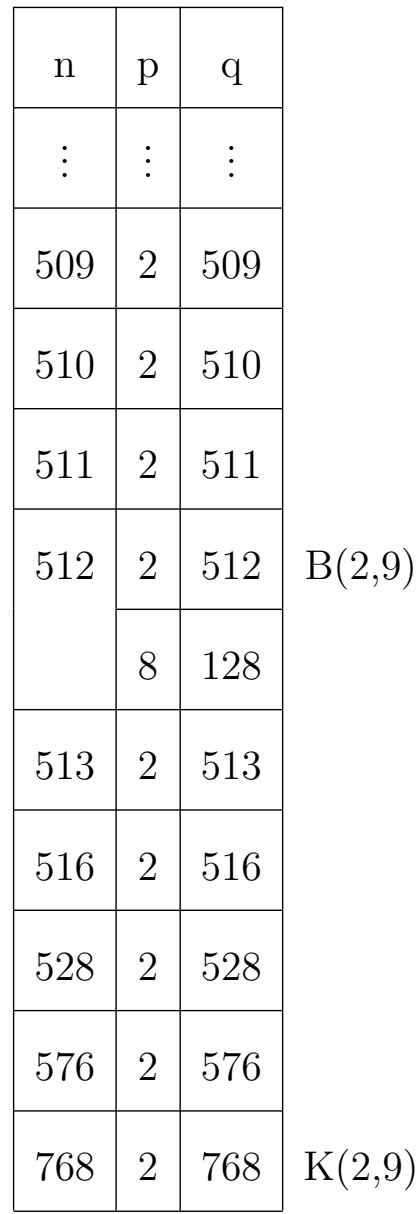

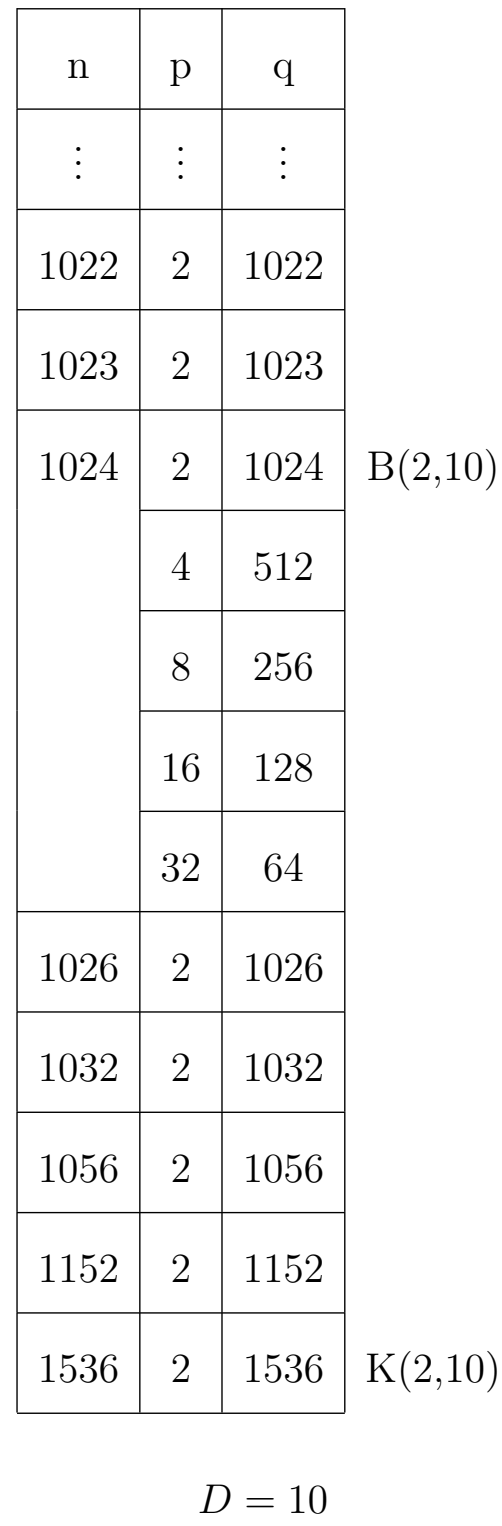

Table 1: $H(p, q, 2)$ with diameters 8,9 and 10. 


\subsection{Efficient $O T I S(p, q)$-layout}

In this section we characterize OTIS $(p, q)$-layouts of de Bruijn digraphs having a minimum number of lenses. For this, we will study cases where $p=d^{p^{\prime}}$ and $q=d^{q^{\prime}}$, as indicated in the discussion above.

Proposition 4.3 The digraph $H(p, q, d)$ is isomorphic to $A\left(f, \mathcal{C}, p^{\prime}-1\right)$, where $\mathcal{C}$ is the complement permutation defined in Definition 2.1, and where the permutation $f$ is defined on $Z_{D}, D=p^{\prime}+q^{\prime}-1$, as follows:

$$
f: i \longmapsto \begin{cases}i+p^{\prime} & \text { if } i<q^{\prime}-1 ; \\ p^{\prime}-1 & \text { if } i=q^{\prime}-1 ; \\ i+p^{\prime}-1(\bmod D) & \text { otherwise. }\end{cases}
$$

Proof: Notice that in this proof, we associate with a number $x \in Z_{d^{k}}$, its $d$-ary representation, denoted $\omega(x)$ (i.e., $\omega(x)=x_{k-1} \ldots x_{1} x_{0}, x_{i} \in Z_{d}$, such that $x=\sum_{i=0}^{k-1} x_{i} d^{i}$ ).

The OTIS $(p, q)$ architecture connects $p$ groups of $q$ transmitters to $q$ groups of $p$ receivers, such that the transmitter $(i, j), 0 \leq i<p, 0 \leq j<q$, is connected to the receiver $(q-j-$ $1, p-i-1)$. Note the $\omega(i)$ has length $p^{\prime}$ and $\omega(j)$ has length $q^{\prime}$. We also label transceiver $(i, j)$ by $\omega(i) \omega(j)$, that has length $p^{\prime}+q^{\prime}$. As $\omega(q-j-1)=\overline{\omega(j)}$ and $\omega(p-i-1)=\overline{\omega(i)}$, we have that the transmitter $\omega(i) \omega(j)$ is connected to the receiver $\overline{\omega(j) \omega(i)}$.

$H\left(d^{p^{\prime}}, d^{q^{\prime}}, d\right)$ has constant degree $d$ and $n$ vertices. Note that since $d$ divides both $p$ and $q$, each group of $d^{q^{\prime}}$ transmitters contains $d^{q^{\prime}-1}$ vertices. So we can label the $k$-th vertex $\left(k \in Z_{d^{q^{\prime}-1}}\right)$ of the $i$-th group of transmitters $\left(i \in Z_{p}\right)$ by $\omega(i) \omega(k)$, of length $p^{\prime}+q^{\prime}-1$. This vertex has the transmitters $\left\{(i, d k+\beta), \beta \in Z_{d}\right\}$, which are labeled by the set of strings $\left\{\omega(i) \omega(k) \omega(\beta), \beta \in Z_{d}\right\}$. Thus, the transmitters of the vertex $\omega(i) \omega(k)$ are connected to 
the receivers $\left\{\overline{\omega(k) \omega(\beta) \omega(i)}, \beta \in Z_{d}\right\}$. Let $\omega(i)=\omega(l) \omega(\alpha)$, where $\omega(l)$ is a string of length $p^{\prime}-1$ and $\alpha \in Z_{d}$. We have $\left\{\overline{\omega(k) \omega(\beta) \omega(i)}, \beta \in Z_{d}\right\}=\left\{\overline{\omega(k) \omega(\beta) \omega(l) \omega(\alpha)}, \beta \in Z_{d}\right\}$ and these receivers belong to the vertices $\left\{\overline{\omega(k) \omega(\beta) \omega(l)}, \beta \in Z_{d}\right\}$.

Finally, a vertex $x$ represented by the string $\omega(x)=\omega(l) \omega(\alpha) \omega(k)$, with $\omega(l) \in Z_{d}^{p^{\prime}-1}$, $\omega(\alpha) \in Z_{d}$ and $\omega(k) \in Z_{d}^{q^{\prime}-1}$, is connected through the OTIS $(p, q)$ architecture, to the vertices represented by the strings $\left\{\overline{\omega(k) \omega(\beta) \omega(l)}, \beta \in Z_{d}\right\}$. As $\omega(x)=x_{D-1} \ldots x_{1} x_{0}$, $x_{i} \in Z_{d}$, we have $\omega(l)=x_{D-1} x_{D-2} \ldots x_{q^{\prime}}, \omega(\alpha)=x_{q^{\prime}-1}$, and $\omega(k)=x_{q^{\prime}-2} \ldots x_{1} x_{0}$. Thus, the digraph $H\left(d^{p^{\prime}}, d^{q^{\prime}}, d\right)$ can be defined on the vertex set $Z_{d}^{D}$ with

$$
\Gamma_{H}^{+}\left(x_{D-1} \ldots x_{1} x_{0}\right)=\left\{\overline{x_{q^{\prime}-2} \ldots x_{0} \beta x_{D-1} \ldots x_{q^{\prime}}}\right\}
$$

According to the definition of $f$ this is exactly $A\left(f, \mathcal{C}, p^{\prime}-1\right)$.

The following lemma allows us to characterize all OTIS $(p, q)$-layouts for de Bruijn digraphs $B(d, D)$.

Lemma 4.4 Let $p^{\prime}+q^{\prime}-1=D$. For any degree $d$, the digraphs $B(d, D)$ and $H\left(d^{p^{\prime}}, d^{q^{\prime}}, d\right)$ are isomorphic if and only if the permutation $f$ of $Z_{D}$ defined by

$$
f: i \longmapsto \begin{cases}i+p^{\prime} & \text { if } i<q^{\prime}-1 ; \\ p^{\prime}-1 & \text { if } i=q^{\prime}-1 ; \\ i+p^{\prime}-1(\bmod D) & \text { otherwise }\end{cases}
$$

is cyclic.

Proof: We showed in Proposition 4.3 that $H\left(d^{p^{\prime}}, d^{q^{\prime}}, d\right)$ is isomorphic to the alphabet digraph $A\left(f, \mathcal{C}, p^{\prime}-1\right)$. We have also shown in Theorem 3.8 that the alphabet digraph 
$A(f, \mathcal{C}, j)$ of degree $d$ and dimension $D$ is isomorphic to the de Bruijn digraph $B(d, D)$ if and only if $f$ is cyclic. The lemma follows.

Now, we show that there exist $p^{\prime}$ and $q^{\prime}$ such that $B(d, D)$ and $H\left(d^{p^{\prime}}, d^{q^{\prime}}, d\right)$ are isomorphic and $d^{p^{\prime}}+d^{q^{\prime}}=\Theta\left(\sqrt{d^{D+1}}\right)$ (i.e., $p+q=\Theta(\sqrt{d n})$, since $\left.d n=d^{D+1}\right)$.

Lemma 4.5 Any OTIS $(p, q)$-layout for $B(d, D)$ has $\Omega(\sqrt{d n})$ lenses.

Proof: Since $p q=d^{D+1}$, we have $p+q \geq 2 \sqrt{d^{D+1}}=2 \sqrt{d n}=\Omega(\sqrt{d n})$.

Proposition 4.6 Let $p^{\prime}=q^{\prime}=\frac{D+1}{2}$. Then $B(d, D)$ and $H\left(d^{\frac{D+1}{2}}, d^{\frac{D+1}{2}}, d\right)$ are isomorphic if and only if $D=1$.

Proof: If $p^{\prime}=q^{\prime}>1, f\left(p^{\prime}-1\right)=q^{\prime}-1=p^{\prime}-1$ and $f$ is not cyclic. If $p^{\prime}=q^{\prime}=1, D=1$ and $B(d, 1)$ is isomorphic to $H(d, d, d)$.

Proposition 4.7 Let $D$ be even, $p^{\prime}=\frac{D}{2}$ and $q^{\prime}=\frac{D}{2}+1$. Then $B(d, D)$ and $H\left(d^{\frac{D}{2}}, d^{\frac{D}{2}+1}, d\right)$ are isomorphic, and therefore $B(d, D)$ has an OTIS $(p, q)$-layout with $p+q=\Theta(\sqrt{d n})$ lenses.

Proof: The permutation $f$ on $Z_{D}$ is defined by

$$
f: i \longmapsto \begin{cases}i+\frac{D}{2} & \text { if } i<\frac{D}{2} ; \\ \frac{D}{2}-1 & \text { if } i=\frac{D}{2} \\ i+\frac{D}{2}-1(\bmod D) & \text { otherwise }\end{cases}
$$

and one can easily verify that $f$ is cyclic. Thus, using Lemma 4.4, the isomorphism is verified.

Furthermore, the $\operatorname{OTIS}(p, q)$-layout has $d^{\frac{D}{2}}+d^{\frac{D}{2}+1}=O(\sqrt{d n})$ lenses, and thus, by Lemma 4.5, the layout has $\Theta(\sqrt{d n})$ lenses. 
Example: Figure 7 shows the digraph $H(4,8,2)$ which has 16 vertices of degree 2 and 32 arcs. The vertex set of $H(4,8,2)$ is $Z_{2}^{4}$, and $\Gamma_{H}^{+}\left(x_{3} x_{2} x_{1} x_{0}\right)=\left\{\overline{x_{1} x_{0} \beta x_{3}}\right\}$. We represent each vertex twice (once at the left of the figure and once at the right), and the arcs going from the left to the right. Figure 8 represents the digraph $B(2,4)$ with the vertices labeling of $H(4,8,2)$.

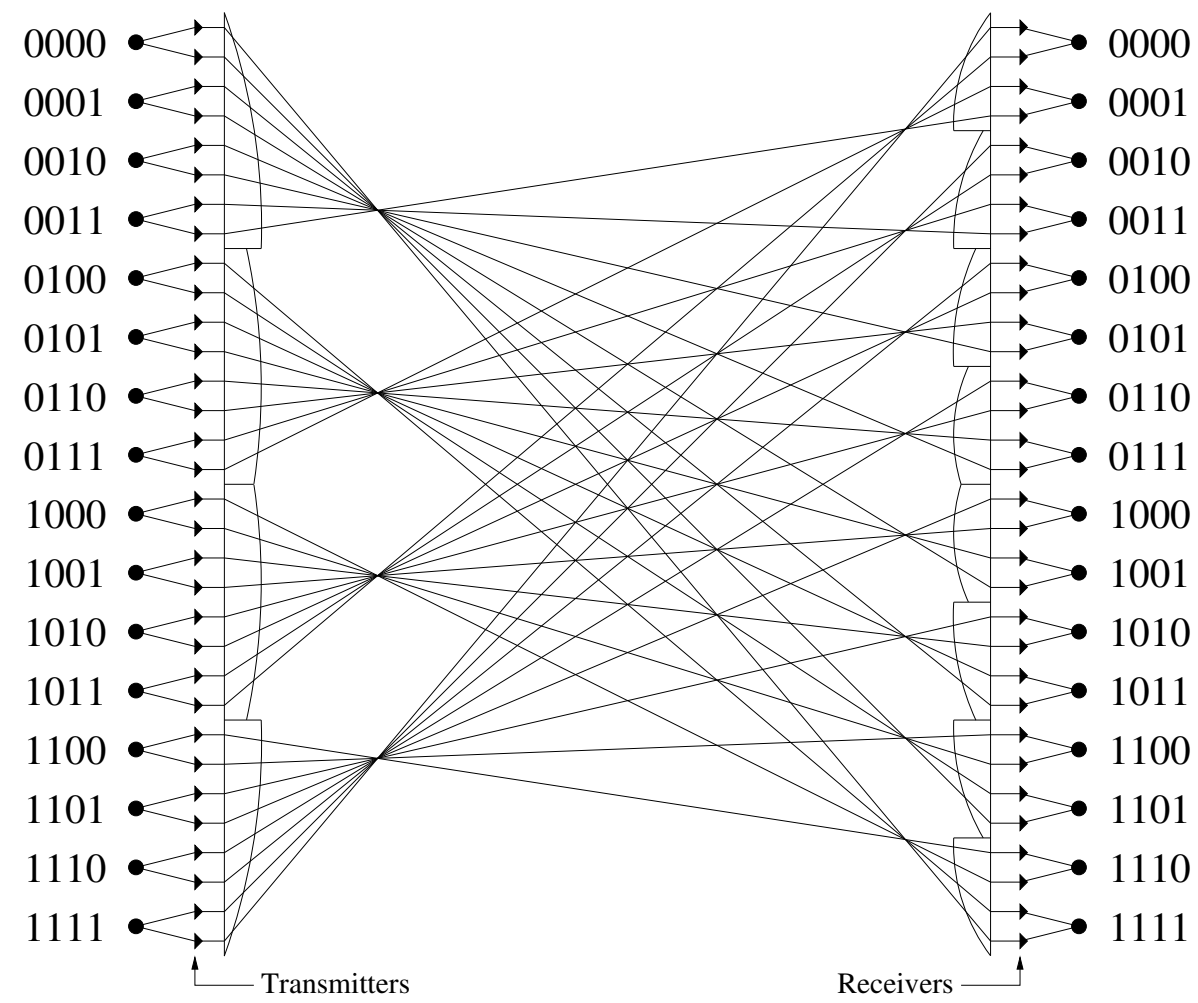

Figure 7: $H(4,8,2)$.

When the diameter $D>1$ is odd, by Proposition 4.6, $B(d, D)$ and $H(p, q, d)$ cannot be isomorphic with $p=q$. The case where $p$ is closest to $q$ is hence when, w.l.o.g., $p^{\prime}=\frac{D-1}{2}$ and $q^{\prime}=\frac{D-1}{2}+2$. However, it is not always the case that $B(d, D)$ and $H\left(d^{\frac{D-1}{2}}, d^{\frac{D-1}{2}+2}, d\right)$ are isomorphic. For instance, $H\left(2^{5}, 2^{7}, 2\right)$ and $B(2,11)$ are isomorphic, while $H\left(d^{6}, d^{8}, d\right)$ and $B(d, 13)$ are not. 


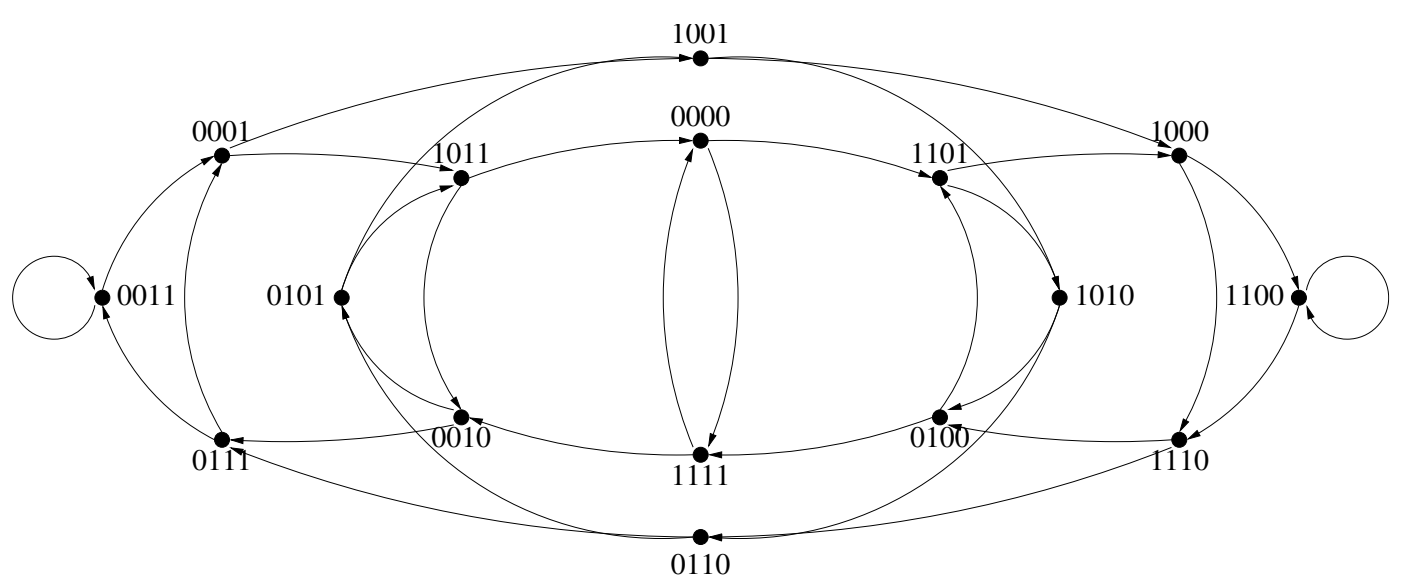

Figure 8: $B(2,4)$ with the adjacency relation of $H(4,8,2)$, i.e.: $\Gamma_{H}^{+}\left(x_{3} x_{2} x_{1} x_{0}\right)=\left\{\overline{x_{1} x_{0} \beta x_{3}}\right\}$.

Corollary 4.8 Given $B(d, D)$ and $H(p, q, d)$, their isomorphism can be verified in $O(D)$ time.

Proof: Using Lemma 4.4 it is sufficient to verify that $f$ is a cyclic permutation on $Z_{D}$. This requires $O(D)$ steps.

Corollary 4.9 Minimizing $d^{p^{\prime}}+d^{q^{\prime}}$, such that $B(d, D)$ and $H\left(d^{p^{\prime}}, d^{q^{\prime}}, d\right)$ are isomorphic, requires $O\left(D^{2}\right)$ time steps.

Proof: If $D$ is even, using Proposition 4.7, the minimum is obtained with $p^{\prime}=\frac{D}{2}$ and $q^{\prime}=\frac{D}{2}+1$. Otherwise, there are $D$ pairs $\left(p^{\prime}, q^{\prime}\right)$, with $p^{\prime}<q^{\prime}$, such that $D=p^{\prime}+q^{\prime}-1$. Thus, using Corollary 4.8, we obtain the minimum value in $O\left(D^{2}\right)$ time.

\section{Conclusion}

In this paper, we have shown that any alphabet digraph defined on words of a given size, through an arbitrary permutation on the alphabet and an arbitrary permutation on the 
word indices, is isomorphic to the de Bruijn digraph, provided that this latter permutation is cyclic. In other words, we have shown that the alphabet digraph $A(f, \pi, j)$, built by using a permutation $f$ on $Z_{D}$, a permutation $\pi$ on $Z_{d}$, an index $0 \leq j \leq D-1$, and the adjacency relation $\Gamma_{A(f, \pi, j)}^{+}(x)=\left\{y_{D-1} \ldots y_{j+1} \beta y_{j-1} \ldots y_{1} y_{0} \mid \beta \in Z_{d}, y_{i}=\pi\left(x_{f(i)}\right)\right\}$ is isomorphic to $B(d, D)$ if and only if $f$ is cyclic.

As an application of this result, we characterized the OTIS $(p, q)$-layout of the de Bruijn digraph $B(d, D)$ when $p$ and $q$ are powers of $d\left(p=d^{p^{\prime}}, q=d^{q^{\prime}}\right.$ and $\left.p^{\prime}+q^{\prime}-1=D\right)$. In particular, we have shown that when the diameter $D$ is even, the minimum number of lenses is obtained with $p=d^{\frac{D}{2}}$ and $q=d^{\frac{D}{2}+1}$, and that for odd diameter $D$, the minimum can be obtained in time $O\left(D^{2}\right)$. For the cases where $p, q \neq d^{i}$, our exhaustive search on all de Bruijn digraphs with less than $2^{32}$ arcs leads us to conjecture that such layouts do not exist.

\section{Acknowledgements}

We are grateful to the anonymous referees for their thorough reading of the manuscript and very helpful comments, and we also would like to thanks Min-Li Yu for his help to improve the readability of the manuscript.

\section{References}

[1] C. Berge. Graphes. Gauthier-Villars, Paris, 1983.

[2] J-C. Bermond, E. Darrot, O. Delmas, and S. Perennes. Hamiltonian circuits in the directed wrapped butterfly network. Discr App Math 84 (1998), 21-42. 
[3] J-C. Bermond, H.A. Harutyunyan, A. Liestman, and S. Perennes. A note on the dimensionality of modified Knödel graphs. Int J Fond Computer Science, Special Issue on Interconnection Networks, 8 (1997), 109-116.

[4] M. Blume, G. Marsden, P. Marchand, and S. Esener. Optical transpose interconnection system for vertical emitters. In OSA Topical Meeting on Optics in Computing, pages 233-235, OSA, Lake Tahoe, NV, March 1997.

[5] W. Bridges and S. Toueg. On the impossibility of directed Moore graphs. J Comb Theory, Series B, 29 (1980), 339-341.

[6] F. Cao and A. Borchers. Optimal transmission schedules for lightwave networks embedded with de Bruijn graphs. Th Comp Science, 222 (1999), 113-131.

[7] D. Chiarulli, S. Levitan, R. Melhem, J. Teza, and G. Gravenstreter. Partitioned optical passive star (POPS) topologies for multiprocessor interconnection networks with distributed control. IEEE/OSA J Light Tech, 14 (1996), 1601-1612.

[8] O. Collins, S. Dolinar, R. McEliece, and F. Pollara. A VLSI decomposition of the de Bruijn graphs. J ACM, 39 (1992), 931-948.

[9] D. Coudert. Algorithmique et optimisation de réseaux de communications optiques. PhD thesis, Université de Nice Sophia-Antipolis, December 2001.

[10] D. Coudert, A. Ferreira, and X. Muñoz. A multihop multi-OPS optical interconnection network. IEEE/OSA J Light Tech, 18 (2000), 2076-2085. 
[11] D. Coudert, A. Ferreira, and X. Muñoz. Topologies for optical interconnection networks based on the optical transpose interconnection system. OSA Appl Opt, 39 (2000), 29652974.

[12] J. de Rumeur. Communications dans les réseaux de processeurs. collection ERI, Masson, Paris, 1994.

[13] M. Feldman, S. Esener, C. Guest, and S. Lee. Comparison between electrical and freespace optical interconnects based on power and speed considerations. OSA Appl Opt, 27 (1988), 1742-1751.

[14] M. Imase and M. Itoh. Design to minimize diameter on building-block network. IEEE Tr Computers, 30 (1981), 439-442.

[15] M. Imase and M. Itoh. A design for directed graphs with minimum diameter. IEEE Tr Computers, 32 (1983), 782-784.

[16] W. Kautz. Bounds on directed $(d, k)$ graphs. Theory of cellular logic networks and machines. AFCRL-68-0668, SRI Project 7258, Final report, pages 20-28, 1968.

[17] G. Marsden, P. Marchand, P. Harvey, and S. Esener. Optical transpose interconnection system architectures. OSA Opt Let, 18 (1993), 1083-1085.

[18] B. Mukherjee. Optical Communication Networks. Series on Computer Communications. McGraw-Hill, New York, 1997.

[19] S. Reddy, D. Pradhan, and J. Kuhl. Directed graphs with minimal diameter and maximal connectivity. Technical report, Oakland University, School of Engineering, 1980. 
[20] S. Reddy, P. Raghavan, and J. Kuhl. A class of graphs for processor interconnection. In H. J. Siegel and L. J. Siegel, editors, Int Conf Parallel Processing, IEEE Computer Society Press, New York, NY, pages 154-157, 1983.

[21] M. Syska. Communications dans les architectures à mémoire distribuée. $\mathrm{PhD}$ thesis, Université de Nice Sophia-Antipolis, 1992.

[22] G. Yayla, P. Marchand, and S. Esener. Energy requirement and speed analysis of electrical and free-space optical digital interconnections. In P. Berthomé and A. Ferreira, editors, Optical Interconnections and Parallel Processing: Trends at the Interface, pages 49-128. Kluwer Academic, Boston, 1997.

[23] F. Zane, P. Marchand, R. Paturi, and S. Esener. Scalable network architectures using the optical transpose interconnection system (OTIS). J Par Distr Computing, 60 (2000), 521-538. 